\title{
Survival and death-promoting events after transient spinal cord ischemia in rabbits: Induction of Akt and caspase3 in motor neurons
}

\author{
Masahiro Sakurai, MD, $\mathrm{PhD}^{\mathrm{a}}$ \\ Tatsuya Nagata, MD, $\mathrm{PhD}^{\mathrm{b}}$ \\ Koji Abe, MD, PhD ${ }^{\mathrm{c}}$ \\ Takashi Horinouchi, MD, PhD ${ }^{\mathrm{d}}$ \\ Yasuto Itoyama, MD, $\mathrm{PhD}^{\mathrm{b}}$ \\ Koichi Tabayashi, MD, PhD ${ }^{\text {a }}$
}

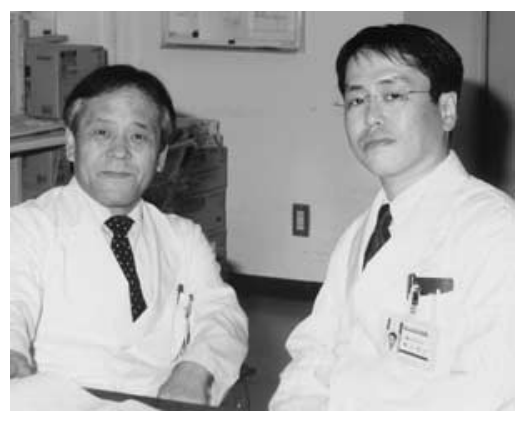

Tabayashi and Sakurai (left to right)
From the Departments of Cardiovascular Surgery, ${ }^{\mathrm{a}}$ Neurology, ${ }^{\mathrm{b}}$ and Anesthesiology, ${ }^{\mathrm{d}}$ Tohoku University Graduate School of Medicine, Sendai, Japan, and Department of Neurology, ${ }^{\mathrm{c}}$ Okayama University Medical School, Okayama, Japan.

Received for publication Dec 26, 2001; revisions requested March 28, 2002; revisions received May 22, 2002; accepted for publication July 8, 2002.

Address for reprints: Masahiro Sakurai, $\mathrm{MD}, \mathrm{PhD}$, Department of Cardiovascular Surgery, Tohoku University Graduate School of Medicine, 1-1 Seiryo-machi, Aoba-ku, Sendai, 980-8574, Japan.

J Thorac Cardiovasc Surg 2003;125:370-7

Copyright $\odot 2003$ by The American Association for Thoracic Surgery

0022-5223/2003 $\$ 30.00+0$

doi: $10.1067 / \mathrm{mtc} .2003 .112$
Objective: The mechanism of spinal cord injury has been thought to be related to the vulnerability of spinal motor neuron cells to ischemia. However, the mechanisms of such vulnerability are not fully understood. We previously reported that spinal motor neurons might be lost as a result of programmed cell death and investigated a possible mechanism of neuronal death by means of immunohistochemical analysis for CPP32 (caspase3) and serine-threonine kinase (Akt).

Methods: We used a rabbit spinal cord ischemia model with use of a balloon catheter. The spinal cord was removed at 8 hours or 1,2, or 7 days after 15 minutes of transient ischemia, and histologic changes were studied with hematoxylin and eosin staining. Western blot analysis for Akt and caspase3, temporal profiles of Akt and caspase 3 immunoreactivity, and double-label fluorescence immunocytochemical studies were performed.

Results: The majority of motor neurons were preserved until 2 days but were selectively lost at 7 days of reperfusion. Western blot analysis revealed no immunoreactivity for Akt and caspase 3 in the sham-operated spinal cords. However, such immunoreactivity became apparent at 8 hours after transient ischemia, decreased at 1 day, and returned to the baseline level at 2 days. A double-label fluorescence immunocytochemical study revealed that both Akt and caspase 3 were positive at 8 hours of reperfusion in the same motor neurons, which eventually die.

Conclusion: These results suggests that transient spinal cord ischemia activates both cell death and survival pathways after ischemia. The activation of Akt protein at the early stage of reperfusion might be one of the factors responsible for the delay in neuronal death after spinal cord ischemia.

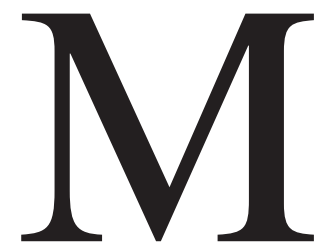

otor neuron dysfunction caused by spinal cord injury after a successful operation of the thoracic aorta is a disastrous complication in human subjects. The reported incidences of paraplegia in such operations range from $2.9 \%$ to $23 \% .^{1}$ The cause of acute spinal cord dysfunction is believed to be ischemic damage during crossclamping. Ischemia can occur because of permanent exclusion of the essential intercostal arterial blood supply to the spinal cord or because of temporary interruption of blood flow to the spinal cord. ${ }^{2}$ However, patients undergoing thoracic aneurysm repair who awake with no neurologic deficit immediately after the operation might sometimes eventually have paraplegia. ${ }^{3,4}$ However, the exact mechanism of such delayed vulnerability is not fully understood. In a rabbit spinal cord ischemia model, we have reported delayed 
and selective motor neuron death after transient ischemia. ${ }^{5,6}$ Furthermore, in this model we have reported that delayed and selective motor neuron death was greatly associated with an activated apoptotic signals of the caspasee 3 cascade. ${ }^{6}$ To evaluate the mechanism of such selective vulnerability of motor neurons, we attempted to make a reproducible model for spinal cord ischemia and analyzed cell damage.

Several reports have suggested that growth factors or neurotrophic factors might play a protective role in brain ischemic injury. ${ }^{7-9}$ Growth factors bind the tyrosine kinase receptors that activate tyrosine phosphorylation. ${ }^{10}$ The subsequent signal cascade is unclear. It has been recently demonstrated that both phosphatidylinositol 3-kinase (PI3-k) and its downstream effector, serine-threonine kinase (Akt), mediate growth factor-induced neuronal survival. ${ }^{11-14}$ For instance, overexpression of activated Akt promoted survival of superior cervical neurons in culture. ${ }^{11}$ Nerve growth factor- or insulin-like growth factor 1-dependent survival of sympathetic or cerebellar neurons was blocked by PI3-k inhibitor, LY294002, and wortmannin. ${ }^{11,13,14}$ Furthermore, activation of the PI3-k/Akt signaling pathway by growth factor suppressed apoptosis and phosphorylation of the bcl-2 family member. Therefore the Akt signaling pathway might be important for neuronal survival.

To understand cell death mechanisms after spinal cord ischemia, we investigated the cell death and survival pathways in motor neurons after transient spinal cord ischemia.

\section{Materials and Methods}

\section{Animal Models}

During the experiment, the animals were treated in accordance with the Declaration of Helsinki and the guiding principles on the care and use of animals. Also, the experimental and animal care protocol was approved by the Animal Care Committee of Tohoku University School of Medicine.

Thirty-three Japanese domesticated white rabbits, weighing approximately 2 to $3 \mathrm{~kg}$, were used in this study and divided into 2 subgroups: a sham control group and a 15-minute ischemia group. Anesthesia was induced by means of intramuscular administration of ketamine at a dose of $50 \mathrm{mg} / \mathrm{kg}$ and maintained with $2 \%$ halothane inhalation. A $5 \mathrm{~F}$ pediatric thermodilution catheter (405, B. Braun Melsungen A.G.) was inserted through a femoral artery and advanced $15 \mathrm{~cm}$ forward into the abdominal aorta. Preliminary investigations confirmed that the balloon in the distal end of the thermodilution catheter was positioned approximately 0.5 to $1.5 \mathrm{~cm}$ just distal to the left renal artery. During the experiment, aortic pressures were continuously monitored both at the proximal and distal positions of the balloon. Body temperature was monitored with a rectal thermometer and was maintained at $37^{\circ} \mathrm{C}$ with the aid of a heating pad during surgical intervention and subsequent ischemia. The animals were then allowed free access to water and food at ambient temperature. In the sham control group animals were killed just after insertion of the catheter into the abdominal aorta, without inflation of the balloon.
TABLE 1. Neurologic score at 2 and 7 days after the procedure

\begin{tabular}{|c|c|c|c|}
\hline \multirow[b]{2}{*}{ Animal no. } & \multirow{2}{*}{$\begin{array}{c}\text { Sham } \\
\text { control, } \\
7 \text { d }\end{array}$} & \multicolumn{2}{|c|}{ 15-min ischemia } \\
\hline & & $2 \mathrm{~d}$ & $7 \mathrm{~d}$ \\
\hline 1 & 5 & 5 & 3 \\
\hline 2 & 5 & 3 & 2 \\
\hline 3 & 5 & 5 & 2 \\
\hline 4 & 5 & 5 & 3 \\
\hline 5 & 5 & 4 & 2 \\
\hline Means $\pm S D$ & $5 \pm 0^{*}$ & $4.9 \pm 0.894 \dagger$ & $2.4 \pm 0.548$ \\
\hline
\end{tabular}

Compared with the 15-minute ischemia group at 7 days after the procedure; ${ }^{*} P=.0090$ and $\dagger P=.0163$.

The animals were divided into 2 experimental groups: group A for investigation by means of histologic study $(n=21)$ and group B for Western blotting and immunohistochemical studies $(\mathrm{n}=12)$.

Group A. Animals were allowed to recover at ambient temperature and were killed by means of deep anesthesia with sodium pentobarbital $(100 \mathrm{mg} / \mathrm{kg}$ administered intravenously) at 8 hours or 1,2 , or 7 days after reperfusion $(\mathrm{n}=$ 3 for 8 hours and 1 day after ischemia, $\mathrm{n}=5$ for sham group and 2 and 7 days after ischemia). Five sham-operated control animals were killed just after the insertion of the catheter into the abdominal aorta, without inflation of the balloon. After death, spinal cords were quickly removed by using the plunger of a 1-mL syringe. The samples for histologic study were fixed by means of immersion in $4 \%$ paraformaldehyde in $0.1 \mathrm{~mol} / \mathrm{L}$ phosphate buffer and then stored at $4^{\circ} \mathrm{C}$ for 1 week; they were then cut transversely at approximately the L2 or L3 level and finally embedded in paraffin.

Group B. For Western blot analysis and immunohistochemical studies, the animals were killed at 8 hours or 1 or 2 days after blood flow restoration $(n=3$ at each time point), and the samples were obtained as noted above for group A. They were kept at $-80^{\circ} \mathrm{C}$ until use. Sham control samples $(n=3)$ were also obtained.

\section{Neurologic Assessment}

Neurologic function was observed at 2 and 7 days after the procedure. Animals were classified by using a 5-point scale according to the method of Johnson and colleagues ${ }^{15}$ : 0, hind-limb paralysis; 1 , severe paraparesis; 2, functional movement, no hop; 3, ataxia, disconjugate hop; 4, minimal ataxia; and 5, normal function. Two individuals without knowledge of the treatment graded neurologic function independently. Statistical analyses of the neurologic scores were done with the Mann-Whitney $U$ test.

\section{Histologic Study}

To see the pathologic changes of the spinal cord after ischemia, we performed hematoxylin and eosin (HE) staining with a set of sections, and examined them by means of light microscopy. The number of intact large motor neuron cells in the ventral gray matter 

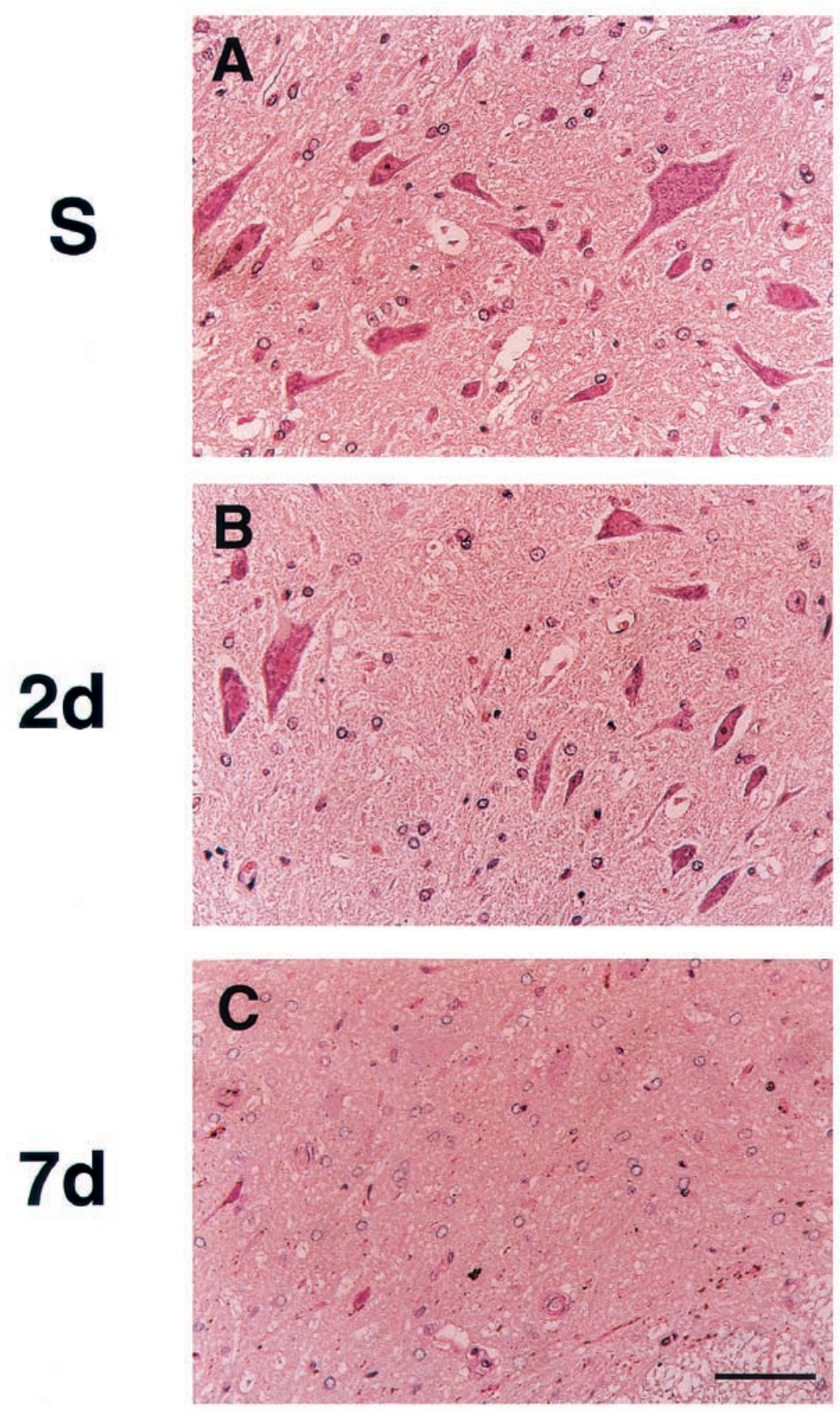

Figure 1. Histologic findings of the spinal cord after 15 minutes of ischemia and staining with HE. Spinal cords of sham control animals $(S ; A)$ and those at 2 days after ischemia (B) showed no histologic changes. At 7 days after ischemia (C), however, motor neurons were selectively lost, without apparent gliosis or cellular infiltration (bar = $100 \mu \mathrm{m})$. S, Sham operated.

region was counted. An observer, unaware of animal group and neurologic outcome, examined each slide. With HE staining, the cells were considered dead if the cytoplasm was diffusely eosinophilic and viable if the cells demonstrated basophilic stippling (ie, contained Nissl substance). ${ }^{16}$ Statistical analysis of the cell number was performed by using the Mann-Whitney $U$ test.

\section{Western Blot Analysis}

To investigate changes of Akt and Caspase 3 expression, we performed Western blot analysis. The tissue samples were homogenized in a lysis buffer $(0.1 \mathrm{~mol} / \mathrm{L} \mathrm{NaCl}, 0.01 \mathrm{~mol} / \mathrm{L}$ Tris- $\mathrm{HCl}[\mathrm{pH}$ 7.5], $1 \mathrm{mmol} / \mathrm{L}$ ethylenediamine tetraacetic acid, and $1 \mu \mathrm{g} / \mathrm{mL}$ aprotinin), and then the homogenates were centrifuged at $7000 \mathrm{~g}$ 
TABLE 2. Numbers of large motor neurons at 2 and 7 days after ischemia

\begin{tabular}{lllc}
\hline Treatment of animals & Date & n & Cell numbers \\
\hline Sham & & 5 & $20.2 \pm 3.033^{*}$ \\
15-min ischemia & 2 d & 5 & $18.4 \pm 5.128 \dagger$ \\
& $7 \mathrm{~d}$ & 5 & $8.4 \pm 2.793$
\end{tabular}

Values are given as means \pm SD.

Compared with the 15-minute ischemia group at 7 days after the procedure; ${ }^{*} P=.0090$ and $\dagger P=.0163$.

for 15 minutes at $4^{\circ} \mathrm{C}$. The supernatants were used as protein samples. Assays to determine the protein concentrations of the supernatants were subsequently performed by means of comparison with a known concentration of bovine serum albumin with a kit (BCA protein assay reagent kit \#23225). Sodium dodecylsulfate (SDS)-polyacrylamide gel electrophoresis was performed in a $10 \%$ polyacrylamide gel under nonreducing conditions. In brief, protein samples were boiled at $100^{\circ} \mathrm{C}$ in $2.5 \% \mathrm{SDS}$ and $5 \%$ $\beta$-mercaptoethanol, and lysates equivalent to $20 \mu \mathrm{g}$ of protein from each samples were run on the gel for 90 minutes at $20 \mathrm{~mA}$, together with a size marker (rainbow-colored protein, Amersham). The electrophoresis running buffer contained $25 \mathrm{mmol} / \mathrm{L}$ Tris base, $250 \mathrm{mmol} / \mathrm{L}$ glycine, and $0.1 \%$ SDS. The proteins on the gel were then transferred to a polyvinylidene fluoride membrane (Micron Separations Inc) with a transfer buffer consisting of $48 \mathrm{mmol} / \mathrm{L}$ Trisbase, $39 \mathrm{mmol} / \mathrm{L}$ glycine, $0.4 \%$ SDS, and $20 \%$ methanol.

After the transfer, the membranes were placed in $1 \%$ powdered milk in phosphate-buffered saline (PBS) to block nonspecific binding. Then they were incubated with primary antibodies at 1:1000 dilution for 20 hours at $4^{\circ} \mathrm{C}$. The primary antibodies used were as follows: goat polyclonal anti-Akt1 antibody (SC-7126; Santa Cruz Biotechnology, Inc) and mouse monoclonal anticaspase3 p20 antibody (SC-1226; Santa Cruz Biotechnology, Inc). After washing in PBS, the membranes were incubated with horseradish peroxidase-anti-goat IgG conjugate (AP180P; Chemicon international, Inc) and horseradish peroxidase-anti-mouse IgG conjugate (AP192P; Chemicon international, Inc) at 1:1000 dilution in PBS for 90 minutes, respectively. The blots were developed with the ECL detection method (RPN2106, Amersham Pharmacia Biotech). Another membrane was stained in a similar way without the primary antibody to ascertain specific binding of the antibody for the protein.

\section{Akt and Caspase3 Immunocytochemistry}

We also performed immunohistochemical study to investigate changes of expression of Akt and caspase3. Spinal cord sections were rinsed in $0.1 \mathrm{~mol} / \mathrm{L}$ PBS for 20 minutes and blocked in $2 \%$ normal horse serum for 2 hours at room temperature. Then they were incubated with primary antibodies in $10 \%$ normal horse serum or $10 \%$ normal rabbit serum and $0.3 \%$ Triton-X 100 for 20 hours at $4{ }^{\circ} \mathrm{C}$, respectively. The primary antibodies used were the same as those used for Western blot analysis noted above, and each dilution was as follows: antibody against Akt at 1:200 and that against caspase 3 at 1:200.

After quenching endogenous peroxidase activity by exposing slides to $0.3 \% \mathrm{H}_{2} \mathrm{O}_{2}$ and $10 \%$ methanol for 20 minutes, the slides were washed in PBS and incubated for 3 hours with biotinylated anti-goat IgG (PK-6105, Vector Laboratories) and biotinylated anti-mouse IgG (PK-6102, Vector Laboratories) at 1:200 dilution in PBS containing $0.018 \%$ normal horse and rabbit serum, respectively. Subsequently, they were incubated with avidin-biotinhorseradish peroxidase complex (PK-6102, Vector Laboratories). The slices were colored with $\mathrm{DAB} / \mathrm{H} 2 \mathrm{O} 2$ solution, and cytoplasm was counterstained with HE. A set of sections were stained in a similar way without the primary antibody to ascertain specific binding of antibody for the protein.

\section{Fluorescence Double-Labeling Study for Akt and Caspase3}

Spinal cord sections were prepared as described above. A nonspecific blocking procedure was performed by using $10 \%$ horse serum before application of primary antibodies. Then the sections were incubated with Akt goat polyclonal antibodies 1:100 (Santa Cruz Biotechnology) simultaneously with caspase 3 . These primary antibodies were incubated overnight at $4^{\circ} \mathrm{C}$ and were detected by using donkey anti-mouse IgG linked with TexasRed I:50 (715075-147, Jackson Immunoreseach) and donkey anti-goat IgG linked with fluorescein isothiocyanate I:50 (705-095-151, Jackson Immunoresearch). The slides were mounted in aqueous mounting media with DABCO and were observed with fluorescein microscopy. We also examined the population of Akt- and caspase3expressing cells. Data are presented as the means \pm SD of 3 rabbits.

\section{Results}

When the balloon of the thermodilution catheter was inflated in the abdominal aorta, the systemic blood pressure of the rabbits did not change. The arterial pressure distal to the inflated balloon decreased to near zero, and no pulsation was recorded. On deflation of the balloon, systemic blood pressure of this portion decreased for 15 minutes and then returned to the normal level (data not shown). Spinal cord ischemia was achieved by means of inflation of the balloon so as to stop blood flow to the spinal cord. ${ }^{17,18}$

\section{Neurologic Outcome}

In the sham-operated group $(\mathrm{n}=5)$ all rabbits were normal (grade 5). In the 15-minute ischemia group at 2 days after the procedure $(\mathrm{n}=5), 3(60 \%)$ rabbits were normal (grade 5), 1 (20\%) rabbit had minimal ataxia (grade 4), and 1 (20\%) rabbit had ataxia (grade 3). In the 15-minute ischemia group at 7 days after the procedure $(\mathrm{n}=5), 3(60 \%)$ rabbits did not hop (grade 2), and 2 (40\%) rabbits had ataxia (grade 3 ). There was a significant difference between the Johnson neurologic scores at 2 and 7 days after the procedure in the 15 -minute ischemia group $(P=.0163)$. This difference was similarly marked between the sham control group and the 15-minute ischemia group at 7 days after the procedure $(P=.0090)$. Fifteen minutes of ischemia did affect neuronal function (Table 1). 


\section{$S \quad 8 h \quad 1 d \quad 2 d$}

\section{Akt}

\section{caspase 3 $\beta$-actin}
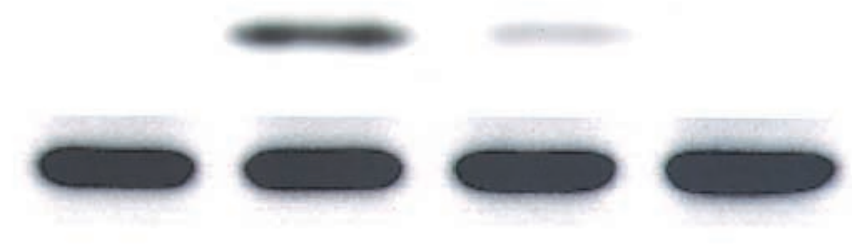

Figure 2. Representative Western blot for Akt (upper), caspase3 (middle), and $\beta$-actin (bottom). Immunoreactivity was not (Akt and caspase3) detected in sham control animals (S). Strong bands became observed at 8 hours (8 h) after blood flow restoration. The band of Akt became scarcely detectable at 1 day; that of caspase 3 became detectable at 1 day after reperfusion and was almost lost at 2 days. $\beta$-Actin showed no change.

\section{Histologic Study}

Representative photographs of HE-stained sections are shown in Figure 1 and Table 2. After sham control, no significant change was seen in motor neurons (Figure 1, A). After 15 minutes of ischemia on the seventh day of reperfusion, approximately $70 \%$ of motor neuron cells were lost (Figure 1,C), although most motor neuron cells had remained intact after 2 days of reperfusion $(P=.0163)$. Small motor neurons and intermediate neurons survived the ischemia (Figure 1, B). Dorsal horn neurons were intact after 15 minutes of ischemia at any time point (data not shown). The results of cell counting in the ventral gray matter region on the paraffin sections obtained from another series of animals are shown in Table 2. The 15-minute ischemic period at 7 days after the procedure affected the number of motor neuron loss cells in contrast to that seen in the sham control animals $(P=.0090)$. Thus selective loss of motor neurons was confirmed, which is in accordance with our previous reports. ${ }^{5,6}$

\section{Western Blot Analysis}

Representative results of Western blot analysis are shown in Figure 2 and Table 3. With antibody against Akt, no band was detectable in samples of sham control, but those at 8 hours after blood flow restoration revealed a single band with a molecular weight of $60 \mathrm{kd}$ (Figure 2, upper panel, and Table 3 ). This band became scarcely detectable at 1 day after reperfusion. With antibody against caspase3, no band was detectable in samples of the sham control, but those at 8 hours after blood flow restoration revealed a single band with a molecular weight of $32 \mathrm{kd}$ (Figure 2, lower panel, and Table 3). This band became detectable at 1 day after reperfusion and was almost lost at 2 days. The membrane
TABLE 3. Signal intensities of Western blot analysis of Akt and caspase 3

\begin{tabular}{|c|c|c|}
\hline & Akt & Caspase3 \\
\hline \multirow[t]{3}{*}{ Sham control } & - & - \\
\hline & - & - \\
\hline & - & - \\
\hline \multirow{3}{*}{$8 \mathrm{~h}$} & $2+$ & $2+$ \\
\hline & $2+$ & $2+$ \\
\hline & $2+$ & $2+$ \\
\hline \multirow[t]{3}{*}{$1 \mathrm{~d}$} & - & \pm \\
\hline & - & \pm \\
\hline & - & \pm \\
\hline \multirow[t]{3}{*}{$2 d$} & - & - \\
\hline & - & - \\
\hline & - & - \\
\hline
\end{tabular}

without the primary antibody revealed no band (data not shown).

\section{Histochemical Study}

Immunoreactive Akt and caspase 3 of sections from spinal cords are shown in Figure 3. The spinal cords of shamoperated animals did not show Akt (Figure 3, A) and caspase3 (Figure 3,E) immunoreactivities in any cells. Motor neurons selectively showed strong immunoreactivity for Akt (Figure 3, B) and caspase 3 (Figure 3, $F$ ) at 8 hours of reperfusion. Immunoreactivity of motor neuron cells for Akt (Figure 3,C) and antigen was almost lost in the motor neurons at 1 day. Immunoreactivity of motor neuron cells for caspase 3 decreased at 1 day (Figure $3, G$ ) and was almost lost in the motor neurons at 2 days (Figure 3, $H$ ).

\section{Fluorescence Double-Labeling Study}

The results of Akt and caspase 3 double-staining immunohistochemistry are shown in Figure 4. Akt was strongly 


\section{Akt}
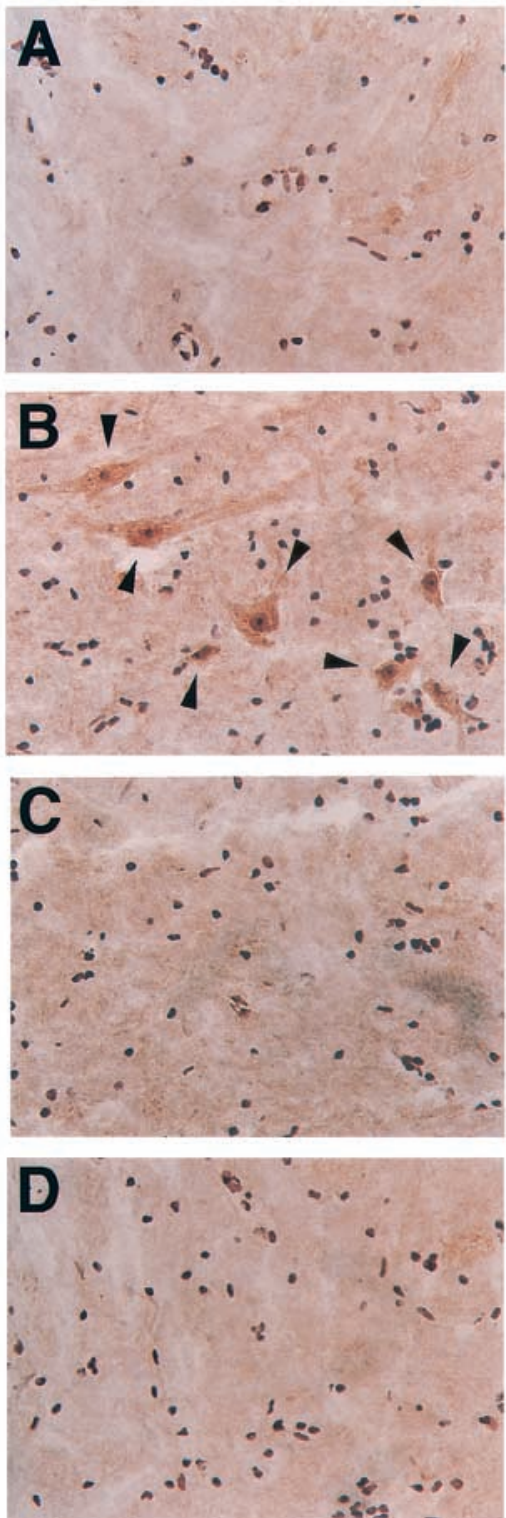

caspase 3
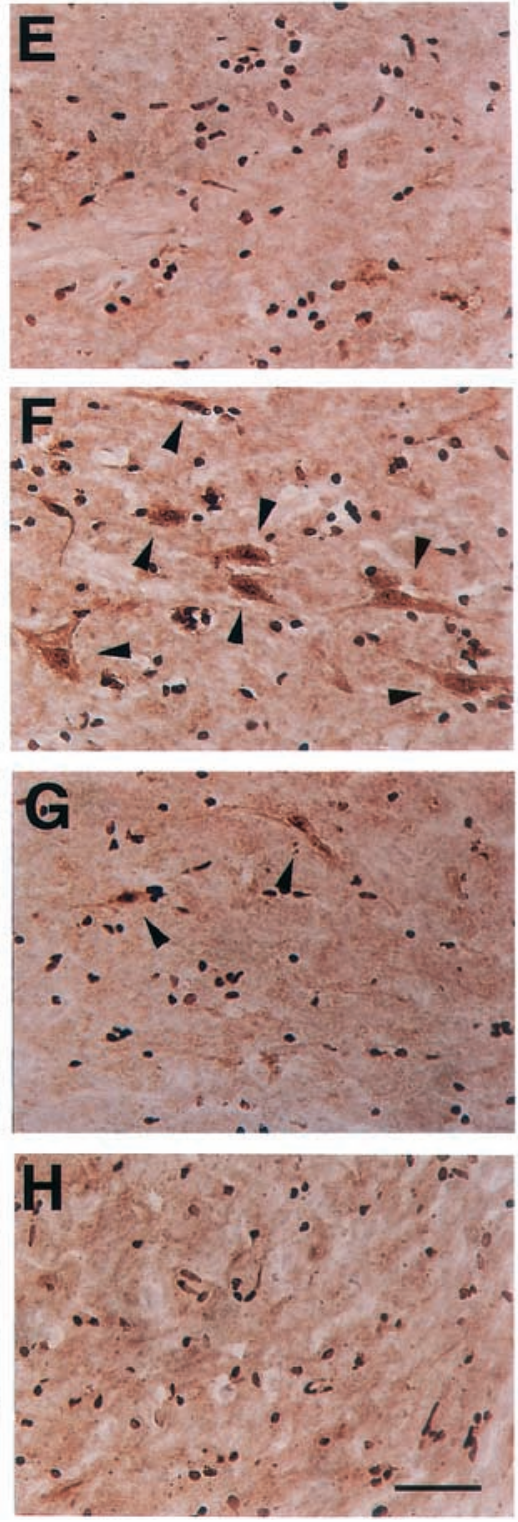

Figure 3. Immunostaining against $A k t$ and caspase 3 in motor neurons in a sham control spinal cord $(A$ and $E)$ and at 8 hours (B and F), 1 day ( $C$ and $G$ ) and 2 days (D and H) of reperfusion. Arrowheads show motor neurons that express immunoreactive Akt (B) and caspase3 (F and G), respectively (bar $=100 \mu \mathrm{m})$. $S$, Sham operated.

colocalized with caspase 3 in motor neurons at only 8 hours of reperfusion. Furthermore, most of the motor neurons expressed both Akt and caspase3 (Table 4).

\section{Discussion}

We have demonstrated delayed and selective motor neuron death in lumbar regions of the rabbit spinal cord with a reproducible model. The histologic patterns of 15 minutes of ischemia in our model are very much reproducible.
Analysis of immunohistochemical study for Akt and caspase 3 were also reproducible at each time point.

We have previously demonstrated delayed and selective motor neuron death in lumbar regions of the rabbit spinal cord with the same reproducible model.5,6 Fifteen minutes of ischemia is a relatively short period in comparison with those of previously reported ischemic models. ${ }^{15}$ After the ischemia, delayed and selective motor neuron damage was observed only after 7 days of reperfusion, a phenomenon 

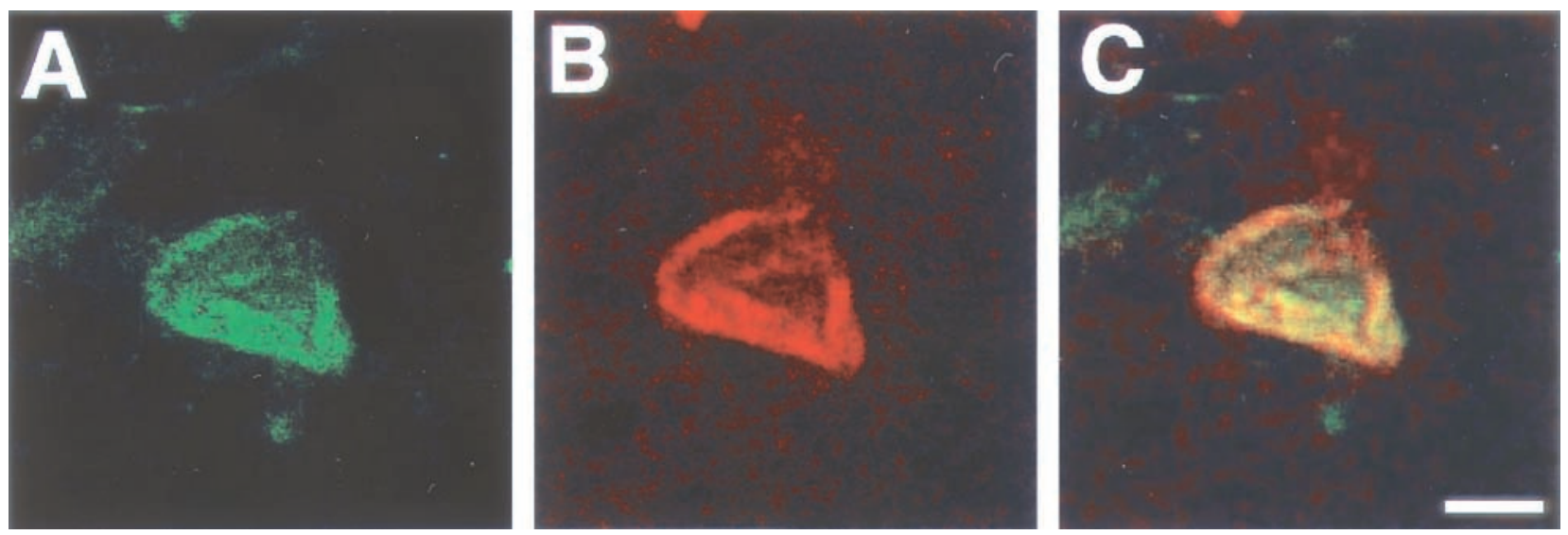

Figure 4. Colocalization of Akt and caspase 3 in motor neurons at 8 hours after ischemia. Akt was detected by means of fluorescein isothiocyanate staining (green; A) and caspase3 by means of Texas red staining (red; B). The merged image is shown in $\mathrm{C}$, with double-positive regions staining a yellow color (bar $=50 \mu \mathrm{m}$ ).

TABLE 4. Colocalization of Akt and caspase3 in spinal motor neurons

No. of motor neurons (mean $\pm \mathrm{SD}$ )

$\begin{array}{ll}\text { Akt positive } & 19.0 \pm 2.6 \\ \text { Caspase3 positive } & 19.3 \pm 2.3 \\ \text { Akt and caspase3 positive } & 15.3 \pm 2.5\end{array}$

known as selective neuronal death in motor neuron cells after spinal cord ischemia, $, 5,6,19$ which is similar to the delayed selective neuronal death in hippocampal CA1 cells after cerebral ischemia. ${ }^{20}$ Despite restoration of blood flow, ${ }^{21}$ motor neurons, which initially appear to have survived ischemic insult, go on to die days later. This result shows that motor neuron cells are most vulnerable to spinal ischemic injury.

Many recent studies on ischemic cerebrovascular disease have revealed that there are 2 major ways for neuronal cell death to occur: necrosis and apoptosis. ${ }^{22}$ Necrosis usually occurs after acute ischemia because of a reduction in the tissue adenosine triphosphate level accompanied by edema formation and concomitant death of glial and vascular cells. In contrast, neuronal apoptosis occurs days after ischemia without a reduction in the adenosine triphosphate level or edema formation and concomitant death of glial and vascular cells. Caspase3 (CPP32) is a member of the interluekinconverting enzyme-like proteases, which are related to mammalian apoptosis and inflammation..$^{23}$ Our previous study showed that an increase in the immunoreactivity of apoptosis-inducing protein, caspase3, was demonstrated in the motor neuron cells of the spinal cord after 15 minutes of transient ischemia, and the peak of caspase 3 induction preceded the DNA fragmentation in the spinal cord after the ischemic insult. ${ }^{6}$ This finding suggests that overexpression of caspase 3 might play an important role in the induction of DNA fragmentation in the spinal cord. The expression of caspase 3 precedes the appearance of neuronal damage and might therefore be implicated in the activation of apoptosis.

In any event some putative protective proteins, such as neurotrophic factors and heat shock protein, might prevent this neuronal death if the proteins can effectively work under emergency conditions, such as ischemia. A recent study has demonstrated that Akt is the target of the PI3-k, which is both necessary and sufficient to mediate inhibition of apoptosis in cerebellar neurons. ${ }^{13}$ PI3-k appears to be a critical component in a signal transduction pathway mechanistically linking viability factors, such as insulin-like growth factor 1, to caspases through activation of Akt. ${ }^{24}$

However, stress-induced activation of PI3-k and Akt has been reported to occur in a number of cell types after treatment with a variety of cellular stresses, such as ischemia. Ouyang and associates ${ }^{25}$ have demonstrated that Akt was activated at an early stage of reperfusion in CA1 neurons of rats, which showed delayed neuronal death. Furthermore, cyt-c was also induced at the same time in the same neurons. Furthermore, Soler and coworkers ${ }^{26}$ have demonstrated that the GDNF family of neurotrophic factors inhibits the activation of caspases through a PI3-k- and Akt-dependent pathway in cultured chicken motor neurons. We have previously shown that giant cell line-derived neutrophilic factor and c-ret proto-oncogene were induced in motor neurons in the same ischemic spinal cord model. ${ }^{27}$ Thus the induction of Akt might indicate a stress response after spinal cord ischemia. We previously showed that PI3-k and Akt would be induced in the rat brain after permanent middle cerebral artery occlusion. ${ }^{28}$ The selective induction of Akt proteins in motor neuron cells might indicate a stress response that occurs in the spinal cord after 15 minutes of ischemia, and almost all motor neurons might be selectively 
damaged after 7 days of reperfusion. Therefore the mechanism of induction of Akt might be similar to the motor neuron cells in the spinal cord and cerebral cortex of the brain.

A recent study showed that the protein levels of PI3-k and Akt were increased significantly in amyotrophic lateral sclerosis (ALS) to that of the control levels. ${ }^{29}$ In other reports they progressively decreased during the presymptomatic stage in transgenic ALS model mice. ${ }^{30}$ In this study the increase in the immunoreactivity of Akt was demonstrated selectively in ventral motor neuron cells in the spinal cord after 8 hours of reperfusion. This finding suggests that the oxidative injury could activate cytokines and augment Akt activity as a compensatory mechanism. Therefore our results suggest that the mechanism of motor neuron death in the spinal cord after ischemia might have features similar to those of ALS.

This study also demonstrates that immunoreactivities for both Akt and caspase 3 were induced at 8 hours in the same motor neuron, which eventually dies. These results suggests that transient spinal cord ischemia activates both cell death and survival pathways after ischemia. The activation of Akt protein at the early stage of reperfusion might be one of the factors responsible for the delay in neuronal death after spinal cord ischemia.

\section{References}

1. Cambria RP, Davison JK, Zannetti S, et al. Clinical experience with epidural cooling for spinal cord protection during thoracic and thoracoabdominal aneurysm repair. J Vasc Surg. 1997;25:234-43.

2. Livesay JJ, Cooley DA, Ventemiglia RA, et al. Surgical experience in descending thoracic aneurysmectomy with and without adjuncts to avoid ischemia. Ann Thorac Surg. 1985;39:37-46.

3. Matsui Y, Goh K, Shinya N, et al. Clinical application of evoked spinal cord potentials elicited by direct stimulation of the cord during temporary occlusion of the thoracic aorta. J Thorac Cardiovasc Surg. 1994;107:1519-27.

4. Moore WM, Hollier LY. The influence of severity of spinal cord ischemia in the etiology of delayed-onset paraplegia. Ann Surg. 1991; 213:427-32.

5. Sakurai M, Hayashi T, Abe K, et al. Cyclin D1 and cdk 4 protein inductions in motor neurons after spinal cord ischemia in rabbits. Stroke. 2000;31:200-7.

6. Hayashi T, Sakurai M, Abe K, et al. Apoptosis of motor neurons with induction of caspases on the spinal cord after ischemia. Stroke. 1998; 29:1007-13

7. Kitagawa H, Hayashi T, Mitsumoto $\mathrm{Y}$, et al. Reduction of ischemic brain injury by topical application of glial cell line-derived neurotrophic factor after permanent middle cerebral artery occlusion in rats. Stroke. 1998;29:1417-22.

8. Schabitz WR, Schwa S, Spranger M, Hacke W. Intraventricular brainderived neurotrophic factor reduces infract size after focal cerebral ischemia in rats. J Cereb Blood Flow Metab. 1997;17:500-6.

9. Tanaka R, Miyasaka Y, Yada K, et al. Basic fibroblast growth factor increase regional cerebral blood flow and reduces infract size after experimental ischemia in a rat model. Stroke. 1995;26:2154-9.
10. Mattson MP, Scheff SW. Endogenous neuroprotection factors and traumatic brain injury: mechanisms of action and implications for therapy. J Neurotrauma. 1994;11:3-33.

11. Crowder RJ, Freeman RS. Phosphatidylinositol 3-kinase and Akt protein kinase are necessary and sufficient for survival of nerve growth factor-dependent sympathetic neurons. J Neurosci. 1998;18: 2933-43.

12. Datta SR, Dudek H, Tao X, et al. Akt phosphorylation of BAD couples survival signals to the cell-intrinsic death machinery. Cell. 1997;91:231-41.

13. Dudek H, Datta SR, Franke TF, et al. Regulation of neuronal survival by the serine-threonine protein kinase Akt. Science. 1997;267:2003-6.

14. Philpott KL, McCarthy MJ, Kippel A, Rubin LL. Activated phosphatidylinositol 3-kinase and Akt kinase promote survival of superior cervical neurons. J Cell Biol. 1997;139:809-15.

15. Johnson SH, Kraimer JM, Graeber GM. Effect of flunarizine on neurological recovery and spinal cord blood flow in experimental spinal cord ischemia in rabbits. Stroke. 1993;24:1547-53.

16. Mutch WAC, Graham MR, Halliday WC, et al. Paraplegia following thoracic aortic cross-clamping in dogs. No difference in neurological out come with a barbiturate versus isoflurane. Stroke. 1993;24:155460.

17. Cheng MK, Robertson C, Grossman RG, et al. Neurological outcome correlated with spinal evoked potentials in a spinal cord ischemia model. J Neurosurg. 1984;60:786-95.

18. Herold JA, Kron IL, Langenburg SE, et al. Complete prevention of postischemic spinal cord injury by means of regional infusion with hypothermic saline and adenosine. J Thorac Cardiovasc Surg. 1994; 107:536-42.

19. DeGirolami U, Zivin JA. Neuropathology of experimental spinal cord ischemia in the rabbit. J Neuropathol Exp Neurol. 1982;41:129-49.

20. Kirino T. Delayed neuronal death in the gerbil hippocampus following ischemia. Brain Res. 1982;239:209-18.

21. Jacobs T P, Kempski O, McKinley D, et al. Blood flow and vascular permeability during motor dysfunction in a rabbit model of spinal cord ischemia. Stroke. 1992;23:367-73.

22. Abe K, Aoki M, Kawagoe J, et al. Ischemic delayed neuronal death: a mitochondrial hypothesis. Stroke. 1995;26:1478-89.

23. Fernandes-Alnemri T, Litwack G, Alnemri ES. CPP32, a novel human apoptotic protein with homology to Caenorhabditis elegans cell death protein Ced-3 and mammalian interleukin- $1 \beta$-converting enzyme. J Biol Chem. 1994;269:30761-4.

24. Cardone MH, Roy N, Stennicke HR, et al. Regulation of cell death protease caspase-9 by phodphorylation. Science. 1998;282:1318-21.

25. Ouyang YB, Tan Y, Comb M, et al. Survival- and death-promoting events after transient cerebral ischemia; phosphorylation of Akt, release of cytochrome $\mathrm{C}$, and activation of caspase-like proteases. J Cereb Blood Flow Metab. 1999;19:1126-35.

26. Soler RM, Dolcet X, Encinas M, et al. Receptors of the glial cell line-derived neurotrophic factor family of neurotrophic factros signal cell survival through the phosphatidylinositol 3-kinase pathway in spinal cord motoneurons. J Neurosci. 1999;19:9160-9.

27. Sakurai M, Hayashi T, Abe K, et al. Inductions of glial cell linederived neurotrophic factor and Ret-like immunoreactivities in rabbit spinal cord after transient ischemia. Neurosci Lett. 1999;276:123-6.

28. Kitagawa H, Warita H, Sasaki C, et al. Immunoreactive Akt, PI3-K and ERK protein kinase expression in ischemic rat brain. Neurosci Lett. 1999;274:45-8.

29. Wagey R, Pelech SL, Duronio V, Kriger C. Phosphatidylinositol 3-Kinase: increased activity and protein level in amyotrophic lateral sclerosis. J Neurochem. 1998;71:716-22.

30. Warita H, Manabe Y, Murakami T, et al. Early decrease of survival signal-related proteins in spinal motor neurons of presymptomatic transgenic mice with a mutant SOD1 gene. Apoptosis. 2001;6:345-52. 